\title{
A Constrained Constant Modulus Algorithm for Proper Polarization Demultiplexing in Coherent Optical Receivers
}

\author{
Omid Zia-Chahabi, Raphaël Le Bidan, Christophe Laot, Michel Morvan \\ Institut Télécom/Télécom Bretagne, UMR CNRS 3192 Lab-STICC \\ Technopôle Brest Iroise CS83818 29238 Brest cedex 3 \\ Université européenne de Bretagne, France \\ omid.ziachahabi@telecom-bretagne.eu
}

\begin{abstract}
We propose a constrained constant modulus algorithm (CMA) for solving the singularity issue of CMA equalizers in polarization-division-multiplexed coherent receivers. Switching to the standard CMA is based on mean square error monitoring over the two output streams.
\end{abstract}

(C) 2011 Optical Society of America

OCIS codes: (060.1660) Coherent communications; (060.4510) Optical communications

\section{Introduction}

Polarization-division multiplexing (PDM), coherent detection and digital signal processing are at the heart of the forthcoming generation of optical transponders for long-haul high-capacity fiber-optic links. Among the various processing done at the receiver are the polarization demultiplexing and equalization of residual chromatic dispersion (CD), polarization-mode dispersion (PMD) and polarization-dependent loss (PDL). To this end, four adaptive finite-impulse response (FIR) filters, arranged in a butterfly structure, are usually used. The blind constant modulus algorithm (CMA) is widely used for updating the filters taps [1]. However, several papers pointed out the singularity issue of the CMA equalizer, whereby the two outputs of the equalizer can converge to the same source when the received signals on both polarizations are in some regions of the Poincaré sphere [2,3]. Several solutions were proposed to overcome this problem, two of which are described in $[2,3]$. These two methods exploit in some way a particular symmetry of the channel transfer matrix induced by the PMD model. In [2], this symmetry is used to initialize the two filters related to the second output after convergence of the first output. In [3], a two-stage approach is used, in which the second stage is a standard CMA whereas the first stage is a symmetrical equalizer which optimizes its taps using one output only.

None of the previous solutions took the symmetry into account in the derivation of the filters weight update equations. The present paper gives the equations obtained from a gradient descent algorithm to minimize the CMA cost function under the constrain of a symmetrical (single-stage) equalizer. In a sense, our constrained CMA resembles the first stage in [3], except that the update equations are optimized from both outputs. We show that this approach is efficient in solving the singularity issue. Finally, since residual CD and PDL break the symmetry of the PMD channel, the constrain on the equalizer has to be released once the mean square error (MSE) on both outputs has reached a given threshold.

\section{Proposed constrained CMA for singularity-free convergence}

When considering PMD as the only transmission impairment, the channel transfer matrix in the frequency domain is unitary [4], i.e.

$$
\mathbf{T}_{P M D}(\omega)=\left(\begin{array}{cc}
u(\omega) & v(\omega) \\
-v^{*}(\omega) & u^{*}(\omega)
\end{array}\right)
$$

where $|u|^{2}+|v|^{2}=1$. In this case, the optimum equalizer is the conjugate transpose of (1) and is also a unitary matrix. In the time-domain, this property translates into the following relationship between the four adaptive filter tap-vectors:

$$
\begin{aligned}
\mathbf{c}_{Y Y}[n] & =\tilde{\mathbf{c}}_{X X}^{*}[n] \\
\mathbf{c}_{Y X}[n] & =-\tilde{\mathbf{c}}_{X Y}^{*}[n]
\end{aligned}
$$


where $(.)^{*}$ stands for the complex conjugate and, for a vector $\mathbf{v}=[v(0), v(1), \ldots, v(N-1)]^{T}, \tilde{\mathbf{v}}$ denotes the timereversed version of $\mathbf{v}$, i.e. $\tilde{\mathbf{v}}=[v(N-1), v(N-2), \ldots, v(0)]^{T}$. Eq. (2) and (3) define the symmetrical and constrained dual-polarization adaptive equalizer.

In contrast to the standard CMA, we now have only two degrees of freedom (e.g. $\mathbf{c}_{X X}$ and $\mathbf{c}_{X Y}$ ) instead of four. We can then use the gradient descent algorithm to obtain the update equations of the constrained CMA equalizer minimizing the cost function:

$$
J_{C M A}\left(\mathbf{c}_{X X}, \mathbf{c}_{X Y}\right)=\mathbb{E}\left[\left(\left|z_{X}[n]\right|^{2}-R\right)^{2}+\left(\left|z_{Y}[n]\right|^{2}-R\right)^{2}\right]
$$

where $z_{X}[n]=\mathbf{c}_{X X}^{T}[n] \mathbf{y}_{X}[n]+\mathbf{c}_{X Y}^{T}[n] \mathbf{y}_{Y}[n]$ and $z_{Y}[n]=\tilde{\mathbf{c}}_{X X}^{*^{T}}[n] \mathbf{y}_{X}[n]-\tilde{\mathbf{c}}_{X Y}^{* T}[n] \mathbf{y}_{Y}[n]$ are the two outputs of the constrained equalizer. Conducting derivations using the rules given in [5] yields:

$$
\begin{aligned}
& \mathbf{c}_{X X}[n+1]=\mathbf{c}_{X X}[n]-\mu\left[z_{X}[n]\left(\left|z_{X}[n]\right|^{2}-R\right) \mathbf{y}_{X}^{*}[n]+z_{Y}^{*}[n]\left(\left|z_{Y}[n]\right|^{2}-R\right) \tilde{\mathbf{y}}_{Y}[n]\right] \\
& \mathbf{c}_{X Y}[n+1]=\mathbf{c}_{X Y}[n]-\mu\left[z_{X}[n]\left(\left|z_{X}[n]\right|^{2}-R\right) \mathbf{y}_{Y}^{*}[n]-z_{Y}^{*}[n]\left(\left|z_{Y}[n]\right|^{2}-R\right) \tilde{\mathbf{y}}_{X}[n]\right]
\end{aligned}
$$

\section{Self-reconfiguring equalizer}

When residual CD and/or PDL are present in addition to PMD, the channel transfer matrix no longer exhibits the symmetry described in Eq. (1). Although the constrained CMA may still be used to solve the CMA singularity issue in such contexts, it will suffer from a performance penalty in steady-state compared to the standard CMA. To overcome this problem, we propose to use a self-reconfigurable equalizer which starts in constrained-CMA mode. The MSE on both polarizations is monitored in real-time and the equalizer eventually switches to standard-CMA once the MSE on both polarizations has reached a predetermined threshold. At the switching instant, the equalizer taps are not optimized for the actual channel but are sufficiently reliable so that the standard CMA will not converge to a singular case.

The estimated MSE on polarization $p$ is computed as:

$$
\operatorname{MSE}_{p}[n+1]=\beta M S E_{p}[n]+(1-\beta)\left(z_{p}[n]-\hat{d}_{p}[n]\right)^{2}
$$

where $\hat{d}_{p}[n]$ is the decided symbol and $\beta$ is a forgetting factor whose aim is to low-pass filter the instantaneous squareerror in order to eliminate its rapid fluctuations.

\section{Simulation results}

Validation of the proposed constrained equalizer is carried out by simulating the setup presented in [2]. A 112Gb/s polarization-multiplexed QPSK signal is impaired by $D=200 \mathrm{ps} / \mathrm{nm}$ of residual CD and $\tau=18 \mathrm{ps}$ of differential group delay (DGD). The received signal has an OSNR $=18 \mathrm{~dB}$ (in a $0.1 \mathrm{~nm}$ noise bandwidth). PDL is modeled as in [6]. Finally, our channel transfer matrix is:

$$
\mathbf{T}(\omega)=\left(\begin{array}{cc}
\cos (\alpha) & e^{-j \phi} \sin (\alpha) \\
-e^{j \phi} \sin (\alpha) & \cos (\alpha)
\end{array}\right)\left(\begin{array}{cc}
e^{j \frac{\omega \tau}{2}} & 0 \\
0 & e^{-j \frac{\omega \tau}{2}}
\end{array}\right)\left(\begin{array}{cc}
\sqrt{1+\gamma} & 0 \\
0 & \sqrt{1-\gamma}
\end{array}\right) e^{j \frac{\lambda^{2} D}{4 \pi c} \omega^{2}}
$$

At the receiver, the $T / 2$-spaced adaptive equalizer is composed of four 7-tap FIR filters and is initialized to $\mathbf{c}_{X X}=$

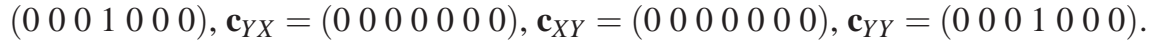

In Fig. 1, rotation angle $\alpha$ was swept from $0^{\circ}$ to $90^{\circ}$ by step of $3^{\circ}$ and phase angle $\phi$ was swept from $-180^{\circ}$ to $180^{\circ}$ by step of $12^{\circ}$ so as to cover the whole Poincaré sphere [2]. Each point was run 100 times with random data and was marked with dark color when at least one singularity issue occured. Fig. 1(a) and 1(b) illustrate the case of polarization demultiplexing using standard CMA with PDL $=1 \mathrm{~dB}$ and $3 \mathrm{~dB}$ respectively. The range of $\alpha$ for which singularity occurs increases with the amount of PDL. Fig. 1(c) shows the case of polarization demultiplexing with the self-reconfiguring equalizer for $\mathrm{PDL}=3 \mathrm{~dB}$. We notice that this new equalizer successfully solves the singularity issue.

Fig. 2(a) compares the convergence and MSE performance of the constrained and standard CMA equalizers (only one polarization is plotted for PDL $=0 \mathrm{~dB}$ ). Convergence speed of the two equalizers is the same. However, due to the CD that breaks the unitary model of the transfer matrix, the constrained equalizer suffers from a steady-state MSE penalty compared to the standard CMA equalizer. For OSNR $=18 \mathrm{~dB}$ and $200 \mathrm{ps} / \mathrm{nm}$ of CD, this penalty is as high as $6 \mathrm{~dB}$. Therefore, the constrained CMA is not suitable for steady-state operation. 


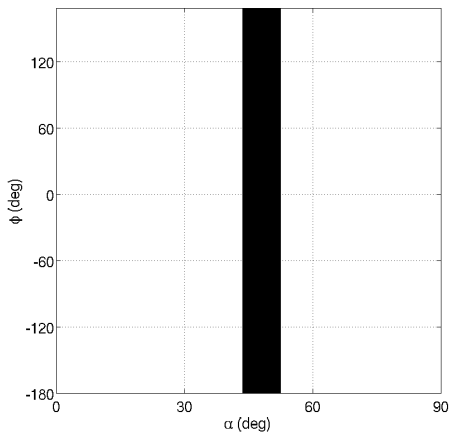

(a)

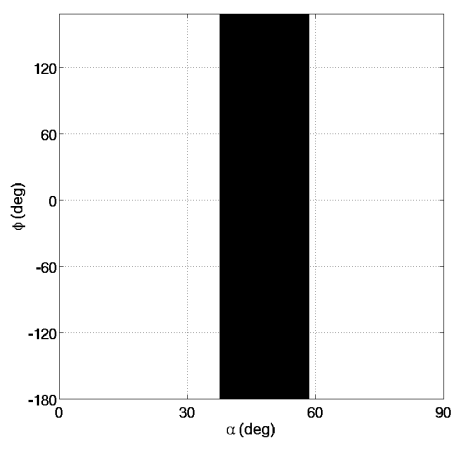

(b)

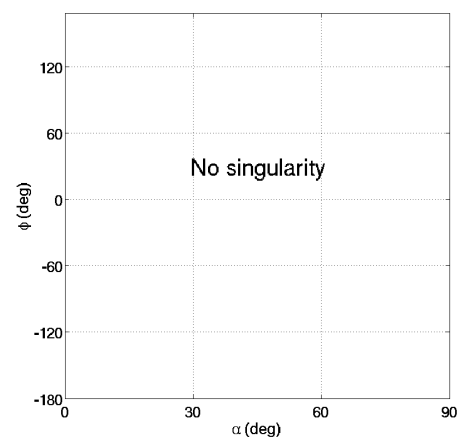

(c)

Fig. 1. Polarization demultiplexing results. (a) Standard CMA, PDL $=1 \mathrm{~dB}$ (b) Standard CMA, PDL $=3 \mathrm{~dB}$ (c) Self-reconfiguring equalizer, $\mathrm{PDL}=3 \mathrm{~dB}$

Fig. 2(b) compares the performance of the self-reconfiguring and standard CMA equalizers. The MSE threshold that triggers switching between constrained and standard mode is set to $-8 \mathrm{~dB}$. The self-reconfiguring equalizer has obviously the same steady-state performance as the standard CMA equalizer, has comparable convergence speed and solves the singularity issue.

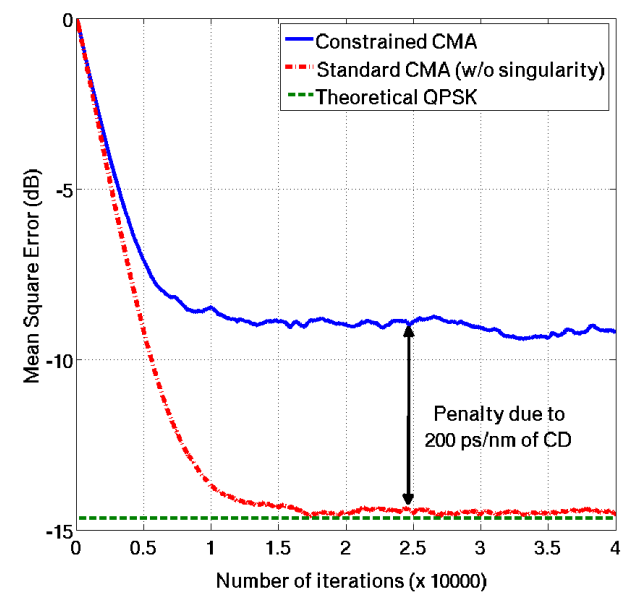

(a)

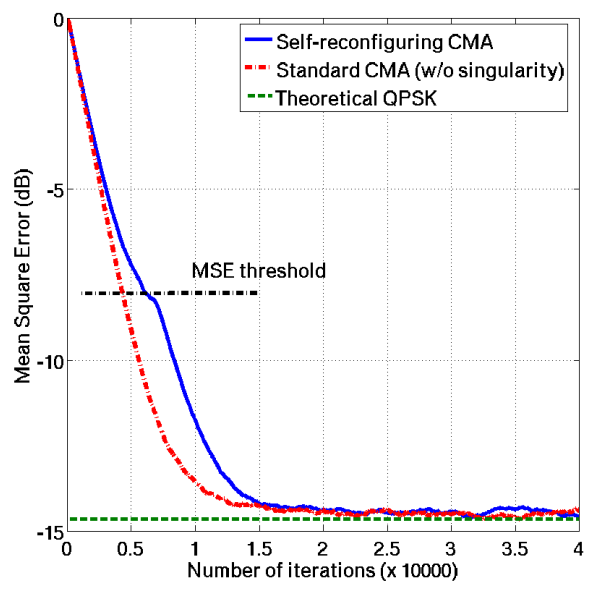

(b)

Fig. 2. Performance comparison between (a) constrained and standard CMA equalizers (b) selfreconfiguring and standard CMA equalizers $(\beta=0.9995)$

\section{Conclusion}

We introduced a constrained CMA equalizer whose convergence is based on a unitary matrix equalizer to solve the singularity problem. Simulations showed that this equalizer is not very tolerant to residual CD and PDL. We proposed a criterion based on MSE monitoring to switch to the standard CMA for steady-state operation.

\section{References}

1. S. J. Savory, Opt. Express, vol. 16, pp. 804-817, 2008.

2. L. Liu, Z. Tao, W. Yan, S. Oda, T. Hoshida, and J. C. Rasmussen, OFC/NFOEC 2009, paper OMT2, 2009.

3. C. Xie and S. Chandrasekhar, OFC/NFOEC 2010, paper OMK3, 2010.

4. C. D. Poole and and R. E. Wagner, Electron. Lett., vol. 22, no. 19, pp. 1029-1030, 1986.

5. A. Hjorungnes, Complex-Matrix Valued Derivatives: With Applications in Signal Processing and Communications, Cambridge University Press, 2011.

6. F. A. Garcia, D. A. Mello, and H. Waldman, Opt. Express, vol. 17, 7958-7969, 2009. 